\title{
ENSAIOS DE IMPACTO IZOD EM COMPÓSITOS POLIMÉRICOS REFORÇADOS COM TECIDO DE RAMI*
}

Noan Tonini SImonassi ${ }^{1}$ Artur Camposo Pereira ${ }^{2}$ Sergio Neves Monteiro ${ }^{3}$

\section{Resumo}

Fibras de rami (Boehmeria nivea) estão entre as lignocelulósicas naturais com potencial para utilização em compósitos poliméricos. O presente trabalho avalia a resistência ao impacto deste tipo de compósito. Corpos de prova normalizados foram confeccionados com percentuais de até 30\% em volume de tecido de rami alinhadas ao longo de todo o comprimento. As fibras foram embutidas sob pressão em matriz de resina poliéster do tipo ortoftálica curada à temperatura ambiente por 24 horas. Os corpos de prova foram ensaiados em pêndulo de impacto Izod e as superfícies de fratura analisadas por microscopia eletrônica de varredura, MEV. A resistência ao impacto aumentou exponencialmente com a quantidade relativa de tecido de rami reforçando o compósito. Este desempenho foi associado à dificuldade de ruptura imposta pelas fibras bem como ao tipo de trincas resultantes da interação tecido de rami/matriz de poliéster que ajuda a absorver a energia do impacto.

Palavras-chave: Rami; Compósitos, Poliéster, Impacto.

\section{IMPACT TESTS IN POLYESTER MATRIX COMPOSITES REINFORCED WITH RAMIE FIBERS}

\begin{abstract}
Ramie fibers (Boehmeria nivea) are among the natural lignocelulósicas potential for incorporation into polymeric composites. This work evaluates the impact resistance of this type of composite. Standard specimens were made with percentages of up to $30 \%$ by volume of ramie cloth. The fibers were embedded under pressure in type orthophtalic polyester resin cured at room temperature for 24 hours. The specimens were tested in an Izod impact pendulum and the fracture was examined by scanning electron microscopy, SEM. The impact resistance increased exponentially with the amount of reinforcing ramie cloth in the composite. This performance can be directly linked to the difficulty of rupture imposed by the fibers and the type of cracks resulting from the interaction of ramie cloth / matrix of polyester that helps to absorb the impact energy.
\end{abstract}

Keywords: Ramie; Composite; Polyester; Impact.

1 Engenheiro Metalurgista, Estudante de Mestrado, Laboratório de fibras Naturais, Instituto Militar de Engenharia (IME), Rio de Janeiro, Brasil.

2 Engenheiro Metalurgista, Estudante de Mestrado, Laboratório de fibras Naturais, Instituto Militar de Engenharia (IME), Rio de Janeiro, Brasil.

3 Engenheiro de Ciências dos Materiais, PhD, Professor titular, Laboratório de fibras Naturais, Instituto Militar de Engenharia (IME), Rio de Janeiro, Brasil. 


\section{INTRODUÇÃO}

Questões ambientais estão assumindo cada vez mais um papel de destaque em nossa sociedade, inclusive condicionando o desenvolvimento tecnológico. $\mathrm{Na}$ engenharia dos materiais, este papel se traduz na maior importância atribuída aos materiais cuja fabricação envolva baixo consumo de energia, alem de poderem ser renováveis e biodegradáveis. Fibras naturais, tanto de origem animal, quanto de origem vegetal lignocelulósicas, atendem estes requisitos. Ou seja, suas utilizações as caracterizam como ambientalmente corretas em comparação com as fibras sintéticas como o náilon, vidro e carbono. Por esta razão, compósitos reforçados com fibras naturais, sobretudo as lignocelulósicas facilmente cultivadas, têm sido objeto de recentes investigações. Um trabalho de revisão sobre este tipo de compósitos foi publicado ao final da última década por Bledzki e Gassan (1). Desde então, centenas de outras publicações foram dedicadas a fibras regularmente cultivadas como a juta ${ }^{(2,3)}$, o linho ${ }^{(4,5)}$, o sisal $(6,7)$, a madeira ${ }^{(8,9)}$, o $\operatorname{coco}^{(10,11)}$ e até mesmo fibra de bananeira $(12,13)$ como exemplos entre muitas outras.

Por outro lado, existe um grupo pouco conhecido de fibras lignocelulósicas que somente agora está sendo investigado. Algumas destas fibras, que também apresentam um grande potencial para uso em compósitos, são típicas de regiões tropicais e, assim, de interesse para países asiáticos, africanos e latino-americanos. No Brasil, exemplos dessas fibras para reforço de compósitos poliméricos são a bucha (14), a piaçava (15), o curauá (16) e o rami (17-21). No caso da fibra de rami, a principal vantagem é a sua resistência à tração, da ordem de $700 \mathrm{MPa}$ (15), comparável à das fibras mais resistentes de sisal e juta. Assim, existe um potencial para se fabricar peças de compósitos, como painéis para construção civil e mobiliário, reforçando a matriz polimérica com tecido de rami.

Estas aplicações exigem que sejam conhecidas as propriedades mecânicas do compósito, em especial sua tenacidade, de importância tanto no processamento quanto no uso de componente fabricados com esse tipo de material. Tendo em vista esses aspectos o objetivo deste trabalho foi avaliar a tenacidade ao entalhe, através de medidas de energia de impacto lzod, de compósitos de matriz poliéster reforçadas com fibras longas e alinhadas de rami.

\section{MATERIAIS E MÉTODOS}

O tecido de rami (Boehmeria nivea) foram adquiridas da firma Sisalsul Indústria de Fibras, que comercializa o produto cultivado no Estado do São Paulo. A análise estatística do lote recebido, efetuada aleatoriamente em 100 fibras, mostrou as distribuições de comprimento e diâmetro na Fig. 1. Destas distribuições obteve-se um comprimento médio de $155 \mathrm{~mm}$ e o diâmetro médio de 0,096 mm. 

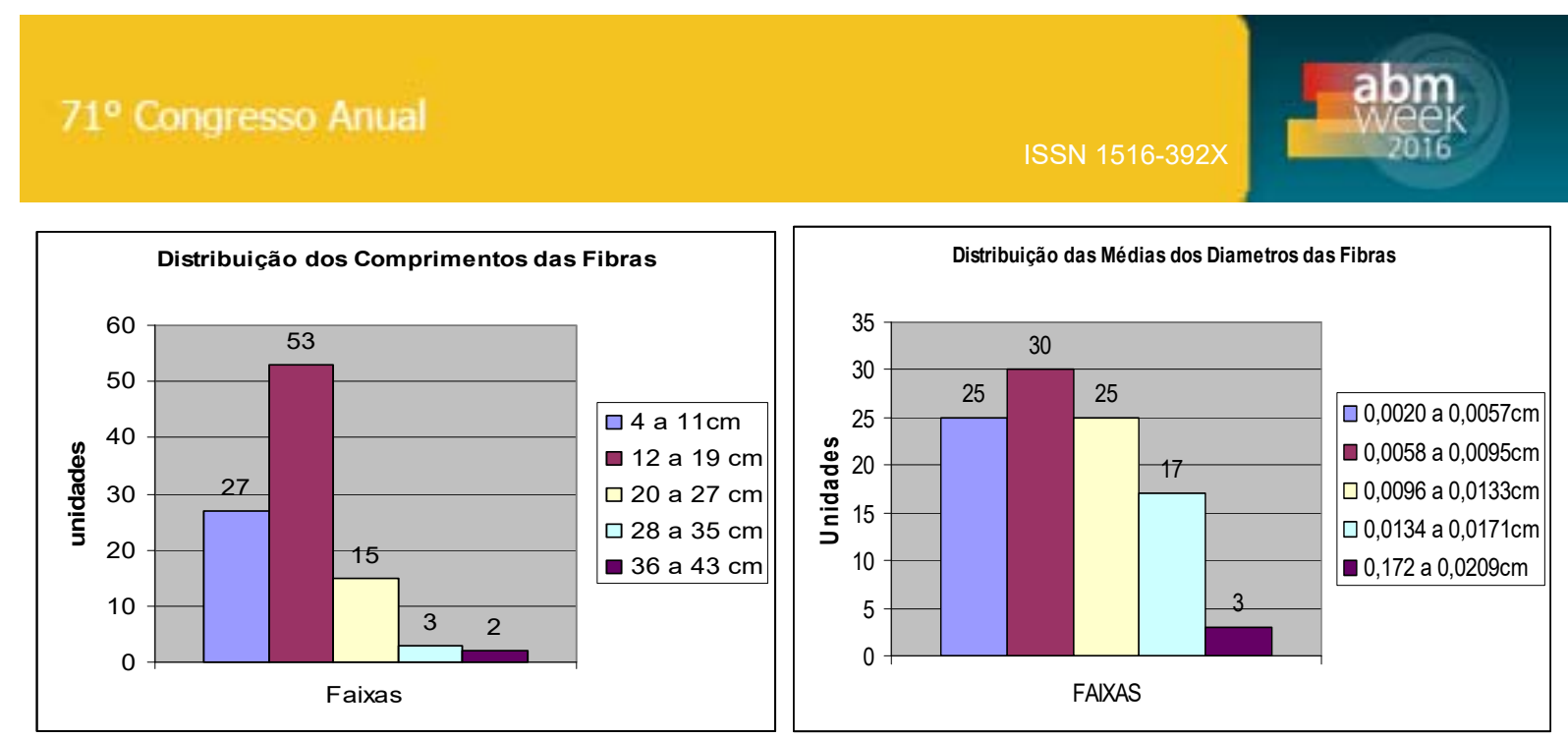

Figura 1: Distribuição do comprimento (a) e do diâmetro (b) da fibra de Rami.

Após separação, limpeza e secagem à temperatura ambiente, as fibras foram misturadas em quantidades de $0,10,20$ e $30 \%$ em volume com resina poliéster ortoftálica para preparar os compósitos. Placas destes compósitos com $10 \mathrm{~mm}$ de espessura foram fabricadas em molde retangular de aço, com dimensões de $152 \mathrm{x}$ $125 \mathrm{~mm}$. As fibras foram mantidas alinhadas ao longo da dimensão de $125 \mathrm{~mm}$.

A resina ainda líquida, acrescida de $0,5 \%$ de catalisador à base de metil-etil cetona, foi vertida sobre o tecido de rami dentro do molde. O compósito assim formado foi deixado curar por 24 horas à temperatura ambiente. As placas de cada compósito foram então cortadas, segundo a direção de alinhamento das fibras, em barras medindo 125 x 12,7 x $10 \mathrm{~mm}$ que serviram como base para confecção de corpos de prova de ensaio de impacto Izod, de acordo com a norma ASTM D256.

Os corpos de prova foram ensaiados em um pêndulo da marca EMIC, em configuração Izod, pertencente ao Instituto Militar de Engenharia. A energia de impacto foi obtida em martelos com potência de $2,7 \mathrm{~J}$, para compósitos de poliéster puro, $0 \%$ de fibra; $5,4 \mathrm{~J}$ para 10 e $20 \%$ de fibra; $10,8 \mathrm{~J}$ para 20 e $30 \%$ de fibra. Para cada condição, 10 corpos de prova foram utilizados e os resultados interpretados estatisticamente.

A superfície de fratura dos corpos de prova após o ensaio de impacto foi analisada por microscopia eletrônica de varredura, MEV, em um equipamento Jeol, modelo JSM-460 LV do Programa de Engenharia Metalúrgica e de Materiais, IME. Amostras cortadas da região de fratura foram metalizadas com ouro e observadas com elétrons secundários acelerados a $15 \mathrm{kV}$.

\section{RESULTADOS E DISCUSSÃO}

Os resultados dos ensaios de impacto em termos de média e de desvio padrão da energia absorvida para cada compósito estão mostrados na Tabela 1.

Tabela 1: Energias de impacto Izod para compósitos poliéster com tecido de rami.

\begin{tabular}{|c|c|}
\hline Percentagem de tecido de Rami & Energia $(\mathrm{J} / \mathrm{m})$ \\
\hline 0 & $15,33 \pm 0,52$ \\
\hline 10 & $84,60 \pm 37,75$ \\
\hline 20 & $463,60 \pm 140,82$ \\
\hline 30 & $537,60 \pm 101,14$ \\
\hline
\end{tabular}

A variação da energia de impacto Izod com a fração em peso do tecido de rami nos compósitos está apresentada na Fig. 2. Nesta figura, observa-se que a incorporação 
de tecido de rami aumenta consideravelmente a tenacidade do compósito. Este aumento, dentro dos limites do desvio padrão, pode ser considerado como exponencial até o máximo de $30 \%$ de fibra. Na realidade o aumento exponencial ocorre dentro de uma extensa faixa indicada pelas linhas do gráfico da Fig 2, passando pelos limites de precisão das barras de erro.

É importante também mencionar que, com o aumento da fração da fibra, aumenta também a dispersão dos valores de tenacidade, traduzindo-se em maiores desvios padrão. Este fato é reconhecido como uma característica das fibras lignocelulósicas (1).

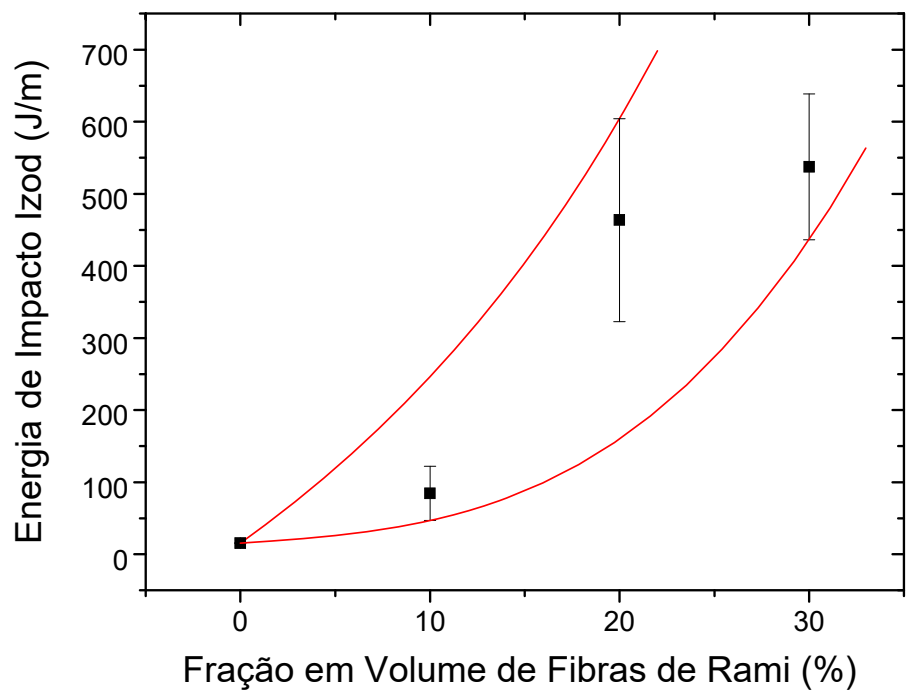

Figura 2: Variação da energia de impacto Izod com a fração de tecido de rami.

A curva mostrada na Fig. 2 é consistente com resultados da literatura, já que o reforço de fibras, tanto sintéticas ${ }^{(22)}$ quanto naturais ${ }^{(15,16)}$ aumenta a tenacidade ao impacto de compósitos com matriz polimérica. A Tabela 2 apresenta valores da tenacidade ao impacto obtida em corpos de prova entalhados para compósitos de matriz polimérica reforçados com fibras naturais.

Tabela 2: Valores de tenacidade ao impacto de fibras naturais.

\begin{tabular}{c|cccc}
\hline Compósito & $\begin{array}{c}\text { Fibra } \\
\text { (\% em peso) }\end{array}$ & Martelo & $\begin{array}{c}\text { Tenacidade ao } \\
\text { Impacto (J/m) }\end{array}$ & Referência \\
\hline Rami/Poliéster & 30 (continua) & Izod & 580 & Presente trabalho \\
Curauá/PP & 50 (picada) & Izod & 54 & $(16)$ \\
Madeira/PP & 50 (picada) & Izod & 28 & $(16)$ \\
Coco/PP & 50 (picada) & Izod & 46 & $(16)$ \\
Linho/PP & 50 (picada) & Izod & 38 & $(16)$ \\
Juta/PP & 50 (picada) & Izod & 39 & $(16)$ \\
Sisal/PP & 50 (picada) & Izod & 51 & $(16)$ \\
\hline
\end{tabular}

No presente trabalho, utilizando-se tecido de rami, os níveis alcançados são bem superiores aos indicados na literatura ${ }^{(16)}$ para tenacidade ao entalhe obtida por impacto Izod em compósitos com matriz de polipropileno, PP, reforçada com $50 \%$ de outras fibras. Obviamente a matriz, PP é diferente da matriz de poliéster usada no presente trabalho, porém podem dar uma idéia comparativa da maior tenacidade de compósitos, quando reforçados com tecido de rami. Em confronto com compósitos de matriz polimérica reforçados com outras fibras naturais picadas (16), a Tabela 2 
mostra que os valores alcançados para compósitos com tecido, tanto de rami na Fig. 2 quanto de piaçava ${ }^{(23)}$, superam em muito todas as outras. A razão para este desempenho pode ser explicada através das características da fratura.

Yue et al (22) sugerem que uma baixa resistência interfacial fibra/matriz contribui para uma ineficiente transferência de esforço da matriz para a fibra, resultando assim em maior energia absorvida no impacto. Por outro lado, existe outro fator que também contribui para a maior tenacidade associada ao reforço de tecido de rami na matriz polimérica. Este fato é uma conseqüência da resistência ao dobramento destas fibras.

A Figura 3 mostra que, macroscopicamente, a incorporação de tecido de rami acarreta um comportamento marcante em relação ao poliéster puro, $0 \%$ de fibra. Mesmo com somente $10 \%$ de fibra, a área de fratura não é totalmente transversal, pois uma parte acompanha o sentido longitudinal de alinhamento das fibras. Isto indica que as trincas geradas no entalhe pelo impacto, iniciam uma propagação transversal pela matriz, como se espera em polímeros, mas ao esbarrarem nas fibras, tendem a se propagar na interface fibra/matriz. Isto está de acordo com o mecanismo de ruptura em compósitos com fraca resistência interfacial (22). Embora pareça paradoxal, uma baixa resistência interfacial acarreta em maior tenacidade, pois gera maior área de fratura e, consequentemente, maior energia absorvida no impacto.

Para frações de tecido de rami de 20 e 30\%, Fig. 3, os corpos de prova nem sequer foram rompidos no impacto. Isto se deve à elevada resistência à tração de tecido de rami, evitando a ruptura total. Para estas maiores frações de fibra, a parte não rompida do corpo de prova dobrou-se o suficiente para permitir que suas extremidades se soltassem dos batentes do equipamento. Neste caso, o resultado da energia de impacto Izod não pode ser comparado com outros em que o corpo de prova é totalmente rompido. Entretanto, a não ocorrência de ruptura no impacto indica uma elevada tenacidade do compósito já que, caso ocorresse a ruptura, a energia absorvida seria ainda superior.

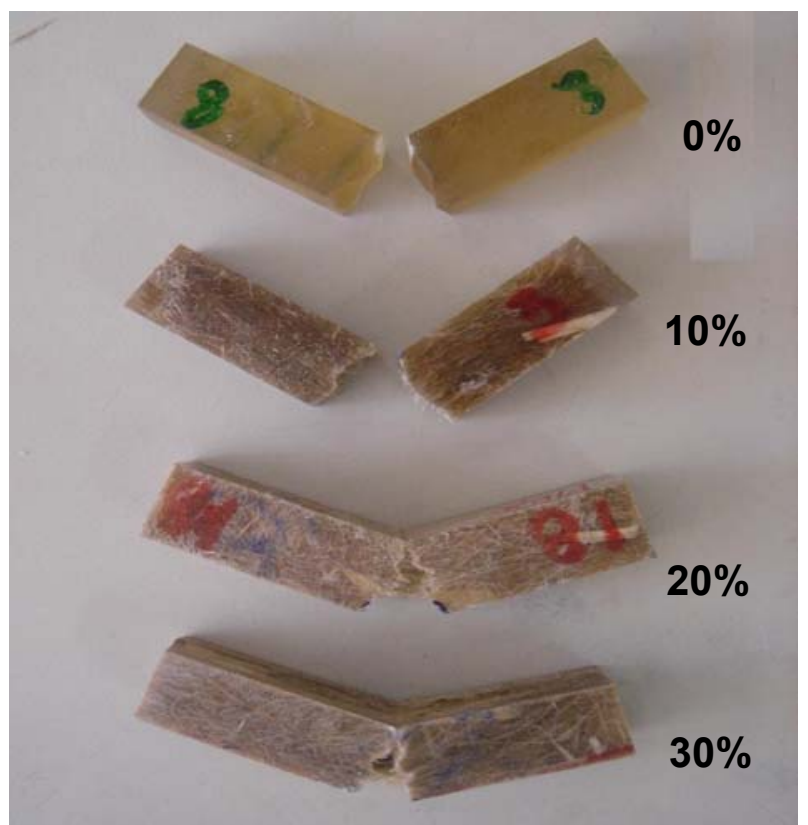

Figura 3: Aspectos macroestruturais da ruptura por impacto Izod de compósitos de matriz poliéster com diferentes frações volumétricas de tecido de rami. 
A análise da microestrutura por MEV na região de fratura resultante do impacto Izod permitiu melhor compreensão dos mecanismos responsáveis pela elevada tenacidade dos compósitos reforçados com tecido de rami.

Em relação à resina pura, Fig.4, o aspecto da fratura é típico do poliéster. Com menor aumento, Fig. 4(a), tem-se uma faixa superior mais marcada e escura, correspondente ao entalhe feito pela fresa. A parte inferior, mais lisa e clara, corresponde à fratura transversal por impacto. Esta fratura, mostrada com maior aumento na Fig. 4(b), revela rugosidade associada à propagação da única trinca, nucleada no entalhe, que ocasionou a ruptura. A trinca acarretou também pequenas cavidades, provavelmente resultantes da porosidade causada pelas condições de processamento.
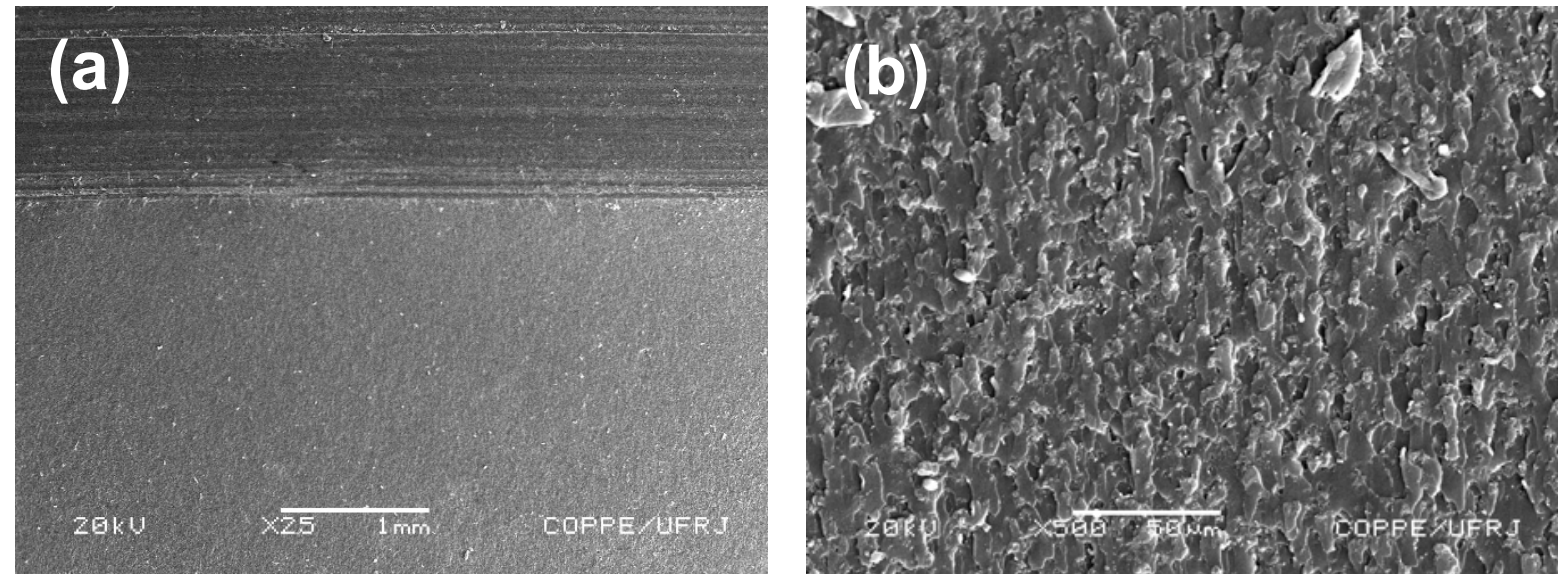

Figura 4: Fratura por impacto do corpo de prova de resina poliéster pura. (a) visão do entalhe e da superfície transversal de ruptura; (b) detalhe da ruptura.

A Figura 5(a), com menor aumento, apresenta por MEV aspectos gerais da superfície rompida por impacto Izod de um corpo de prova com $30 \%$ em volume de fibras de rami reforçando matriz poliéster.
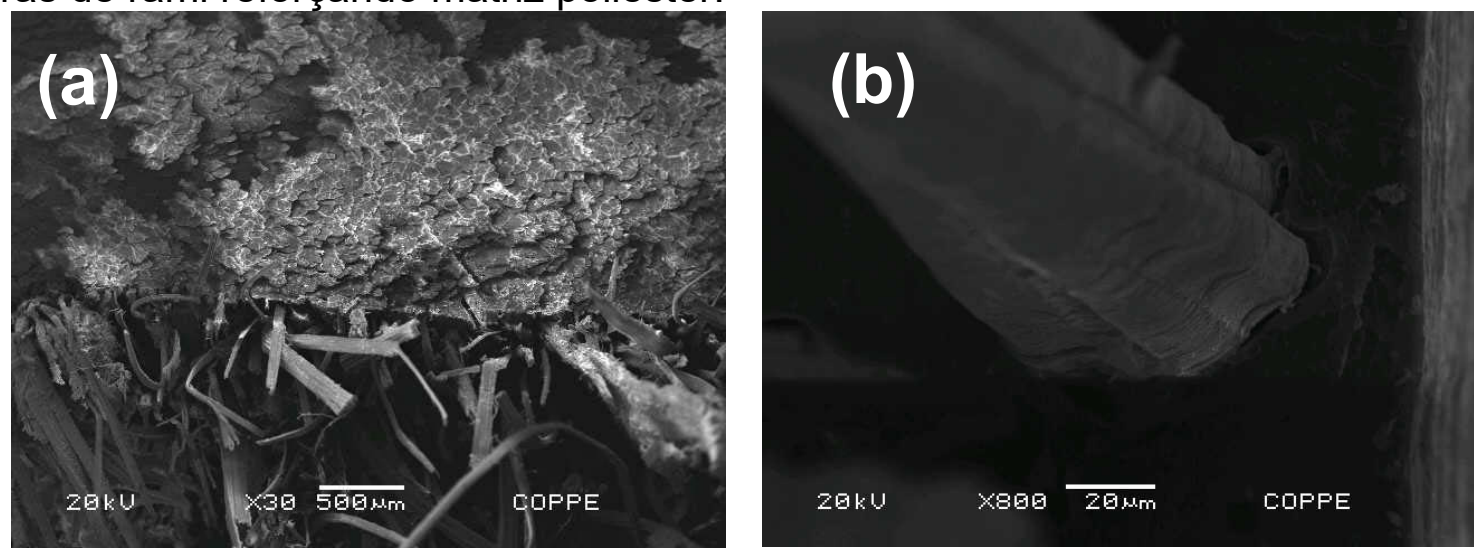

Figura 5: Fratura por impacto do corpo de prova do compósito com 30\% em volume de tecido de rami.

É importante notar que na Fig. 5(b), observada com maior aumento na região em que a fratura propagou-se seguindo a interface fibra/matriz, existem evidências de descolamento interfacial. Este comportamento corrobora o mecanismo de ruptura através de trincas que se propagam preferencialmente entre a fibra e a matriz devido à fraca resistência interfacial (22). A maior área longitudinal de ruptura dos compósitos como consequência do reforço do tecido de rami, justifica a elevada energia absorvida no impacto, bem como seu aumento com a fração de fibra. 


\section{CONCLUSÃO}

Compósitos constituídos de tecido de rami reforçando matriz poliéster, curada à temperatura ambiente, apresentam um aumento exponencial na tenacidade ao entalhe, medida em ensaios de impacto Izod, em relação à resina poliéster pura.

Nestes compósitos, para a fração de fibra incorporada de até $30 \%$ em volume, atingem-se as mais altas tenacidades já registradas em fibras lignocelulósicas. Parte deste aumento na tenacidade é devido à baixa tensão interfacial entre o tecido de rami e a resina poliéster. Isto acarreta maior energia absorvida no impacto em decorrência da propagação de trincas na interface fibra/matriz, gerando maior área de ruptura em relação a uma fratura transversal que ocorra na matriz rompendo as fibras.

Para frações de tecido de rami superiores a $10 \%$ em volume, o aumento da tenacidade ao entalhe pode também, em parte ser devido à energia despendida em se dobrar as fibras que não são rompidas após o impacto do martelo Izod.

\section{REFERÊNCIAS}

1 BLEDZKI, A.K.; GASSAN, J. Composites Reinforced with Cellulose-Based Fibres. Prog. Polym. Sci, v. 24, p. 221-274, 1999.

2 KUMAR, A.P.; SINGH, R.P.; SARWADE, B.D. Degradability of composites prepared from ethylene-polypropylene copolymer and jute fiber under accelerated aging and biotic environments. Mater. Chem. and Phys., v. 92, p. 458-469, 2005.

3 DOAN, T.-T.-L.; GAO, S.-L.; MÄDER,E. Jute/polypropylene composites. I. Effect of matrix modification. Compos. Sci. Technol., v. 66, p. 952-963, 2006.

4 CANTERO, G.; ARBELAIZ, A.; LHANO-PONTE, R.; MONDRAGON, I. Effects of fiber treatment on wettability and mechanical behaviour of flax/polypropylene composite. Compos. Sci. Technol., v. 63, p. 1247-1254, 2003.

$5 \quad$ VAN DE WEYENBERG, I.; IVENS, J.; DE COSTER, A.; KINO, B.; BATENS, E.; VERPOEST, I. Influence of processing and chemical treatment of flax fibres on their composites. Compos. Sci. Technol., v. 63, p. 1241-1246, 2003.

6 JOSEPH, P.V.; JOSEPH, K.; THOMAS, S. Short sisal fiber reinforced polypropylene composites: the role of interface modification on ultimate properties. Compos. Interf., v. 92, n. 2, p. 171-205, 2002.

7 FUNG, K.L.; LI, R.K.Y.; TJONG, S.C. Interface modification on the properties of sisal fiber-reinforced polypropylene composites. J. Appl. Polym. Sci., v. 85, p. 169-176, 2002.

8 ALBANO, C.; ICHAZO, M.; GONZALEZ, J.; DELGADO, M.; PALEO, R. Effects of filler treatments on the mechanical and morphological behavior of PP + wood flour and PP + sisal fiber. Mater. Research Innovat., v. 4, p. 284-293, 2001.

9 CHUAI, C.; ALMDAL, K.; POULSEN, L.; PLACKETT, D. Conifer fibers as reinforcing materials for polypropylene-based composites. J. Appl. Polym. Sci., v.80, p. 28332841, 2001.

10 ROUT, J.; TRIPATHY, S.S.; MISHRA, M.; MOHANTY, A.K; NAYAK, S.K. The influence of fiber surface modification on mechanical properties of coir-polyester composites. Polym. Composites. v. 22, p 468-472, 2001.

11 MONTEIRO, S.N.; TERRONES, L.A.H.; CARVALHO, E.A.; D'ALMEIDA, J.R.M. Efeito de interface fibra/matriz sobre a resistência de compósitos poliméricos reforçados com fibras de coco, Rev. Mater., v. 11, n. 4, p. 395-402, 2006.

12 POTHAN, L.A.; GEORGE, J.; THOMAS, S. Effect of fibre surface treatment on the fibrematrix interaction in banana fibre reinforced polyester composites, Compos. Interf., v. 9, n. 4, p. 335-353, 2002. 
13 POTHAN, L.A.; THOMAS, S. Polarity parameters and dynamic mechanical behaviour of chemical banana fibre reinforced polyester composites, Compos. Sci. Technol., v. 63, p. 1231-1240, 2003.

14 BOYNARD, C.A.; MONTEIRO, S.N.; D'ALMEIDA, J.R.M. Aspects of Alkali Treatment of Sponge Gourd (Luffa cylindrica) Fibers on the Flexural Properties of Polyester Matrix Composites. J. Appl. Polym. Sci. v. 87, p.1927-1932, 2003.

15 SATYANARAYANA, K.G.; GUIMARÃES, J.L.; WYPYCH, F. Studies on lignocellulosic fibers of Brazil. Part I: Source, production, morphology, properties and applications. Composites: Part A, v. 38, p. 1694-1709, 2007.

16 LEÃO, A.L.; TAN, I.H.; CARASCHI, J.C. Curaua Fiber - A Tropical Natural Fibre from Amazon - Potential and Applications in Composites. In: INTERNATIONAL CONFERENCE ON ADVANCED COMPOSITES, Hurghada, Egypt, May, 1998, p. 557564.

17 ANGELINI, L. G.; LAZZERI, A.; LEVITA, G.; FONTANELLI, D.; BOZZI, C. Ramie (Boehmeria nivea (L.) Gaud.) and Spanish Broom (Spartium junceum L.) fibers for composite materials: agronomical aspects, morphology and mechanical properties, Industrial Crops and Products, v.11, n. 2-3, p.145-161, 2000.

18 HINDELEH, A. M.; JOHNSON, D. J.; Crystallinity and crystallite size measurement in cellulose fibres: Ramie and Fortisan. Polymer, v. 13, n. 9, p. 423-430, 1972.

19 SHIHONG, L.; BENLIAN, Z.; QIYUN, Z.; XIANRONG, B., A new kind of super-hybrid composite material for civil use - ramie fibre/Al, Composites, v. 25, n. 3, p. 225-228, 1994.

20 ISHIKAWA, A.; OKANO, T.; SUGIYAMA, S.; Fine structure and tensile properties of ramie fibres in the crystalline form of cellulose I, II, III, and IV. Polymer, v. 38, n.2, p.463468, 1997.

21 WOLLERDORFER, M.; BADER, H.; Influence of natural ramie fibers on the mechanical properties of biodegradable polymers, Industrial Crops and Products, v. 8, n. 2, p. 105112, 1998.

22 YUE, C.Y.; LOOI, H.C; QUEK, M.Y. Assessment of Fibre-Matrix Adhesion and Interfacial Properties Using the Pullout Test. Int. J. Adhesion and Adhesives, v. 15, p. 73-80, 1995.

23 MONTEIRO, S.N.; AQUINO, R.C.M.P.; LOPES, F.P.D.; CARVALHO, E.A.; D'ALMEIDA, J.R.M. Tenacidade ao entalhe por impacto Charpy de compósitos de poliéster reforçados com fibras de piaçava. Rev. Mater., v. 11, n. 3, p. 204-210, 2006. 